\title{
Case of New Onset Acute Leukemia Complicated by Renal Calculi and Gout Attack: A Case Report
}

\author{
Cima Hamieh$^{\mathrm{a}, \mathrm{c}}$, Mahmoud El-Hussein ${ }^{\mathrm{b}}$, Elie Zaghrini ${ }^{\mathrm{b}}$
}

\begin{abstract}
Increased level of uric acid could be due to several etiologies, mainly non-balanced diet, and if uncontrolled can cause serious outcomes. Hyperuricemia's major adverse effects in an individual can include nephropathy, nephrolithiasis and uric acid depositions in joints. On the other hand, hyperuricemia can be the result of several underlying disorders, of which are the hematologic malignancies. A case presented to our institution with generalized fatigue found to have multiple electrolytes abnormalities. On further investigation, the patient was diagnosed with acute leukemia, hyperuricemia and kidney calculus and acute gouty arthritis concurrently, and treated with surgery, corticosteroid and uric acid lowering agents and pain management. Diagnosis and rapid management of acute leukemia associated with hyperuricemia is crucial to avoid the complication of uric acid accumulation in different body organs.
\end{abstract}

Keywords: Acute leukemia; Kidney stone; Acute gouty arthritis; Hyperuricemia

\section{Introduction}

Uric acid, found in meat, seafood and alcoholic beverages, is an antioxidant to the human body if consumed in balanced amounts. A normal serum uric acid level ranges $2.4-6.0 \mathrm{mg} /$ $\mathrm{dL}$ in females and $3.4-7.0 \mathrm{mg} / \mathrm{dL}$ in males. However, higher levels of uric acid cause complications such as kidney stone and gout [1]. Kidney diseases, endocrinopathies, chemotherapy and certain cancers have been identified as secondary causes of hyperuricemia [1].

The cases reported in the literature associating the com-

Manuscript submitted February 2, 2021, accepted March 1, 2021

Published online March 16, 2021

${ }^{a}$ Department of Family Medicine, Lebanese American University Medical Center, Beirut, Lebanon

${ }^{b}$ Department of Emergency Medicine, Lebanese American University Medical Center, Beirut, Lebanon

${ }^{c}$ Corresponding Author: Cima Hamieh, Department of Family Medicine, Lebanese American University Medical Center, Beirut, Lebanon.

Email: Cyma.hamieh@gmail.com

doi: https://doi.org/10.14740/wjnu424 plications of hyperuricemia with leukemia are very rare. One article that dates back to the 1950 s mentions a case of chronic myelogenous leukemia where successive increases in nucleoprotein catabolism precipitated a gouty arthritis attack [2]. Another case links tumor lysis syndrome to an acute gouty attack, few days after percutaneous radiofrequency thermal ablation of hepatocellular carcinoma [3]. No other cases mention an inflammatory attack of a joint in a patient with acute leukemia prior to treatment initiation.

Gout and urate kidney stones are complications of hyperuricemia. Looking at the incidence of these complications in leukemic patients, we found that urate kidney stones are usually associated with chemotherapy initiation, or spontaneous tumor lysis syndrome in lymphoblastic leukemia [4,5]. A case report of a patient with acute myeloid leukemia (AML), 22 months after chemotherapy initiation, found to have a urate kidney stone is described [6].

\section{Case Report}

A 50-year-old male patient presented to our emergency department with progressive generalized fatigue, acute onset of left flank pain and lower extremity edema for the last 2 weeks. On further history, the patient denied any fever, night sweats, weight loss, dyspnea, orthopnea or paroxysmal nocturnal dyspnea (PND). He has a normal appetite, regular bowel movement, no recent respiratory symptoms and no urinary symptoms. He drinks alcohol occasionally and he is a non-smoker. This patient is overweight, known to have hypertension on valsartan $160 \mathrm{mg}$ and hydrochlorothiazide 25 $\mathrm{mg}$ daily, and atrial fibrillation on bisoprolol $5 \mathrm{mg}$ and aspirin daily.

In the emergency department, vitals were all within normal limits. On physical exam, there was no lymphadenopathy with good bilateral airway entry on auscultation and irregular heart rate, his abdomen was soft with regular bowel sounds, and there were no organomegalies palpated. Lower extremity pitting edema was noted.

Laboratory testing was significant for a white cell count of 26,000 , with $14.7 \%$ lymphocytes, $61 \%$ neutrophils, $14 \%$ monocytes and a large unstained cells (LUC) count of $1.2 \%$ with a normal of $0.4 \%$; the patient was also anemic with a hemoglobin of 6.3 and mean corpuscular volume (MCV) of 105. The remainder of the complete blood count was non-significant. On chemistry exam, the patient had a potassium of 

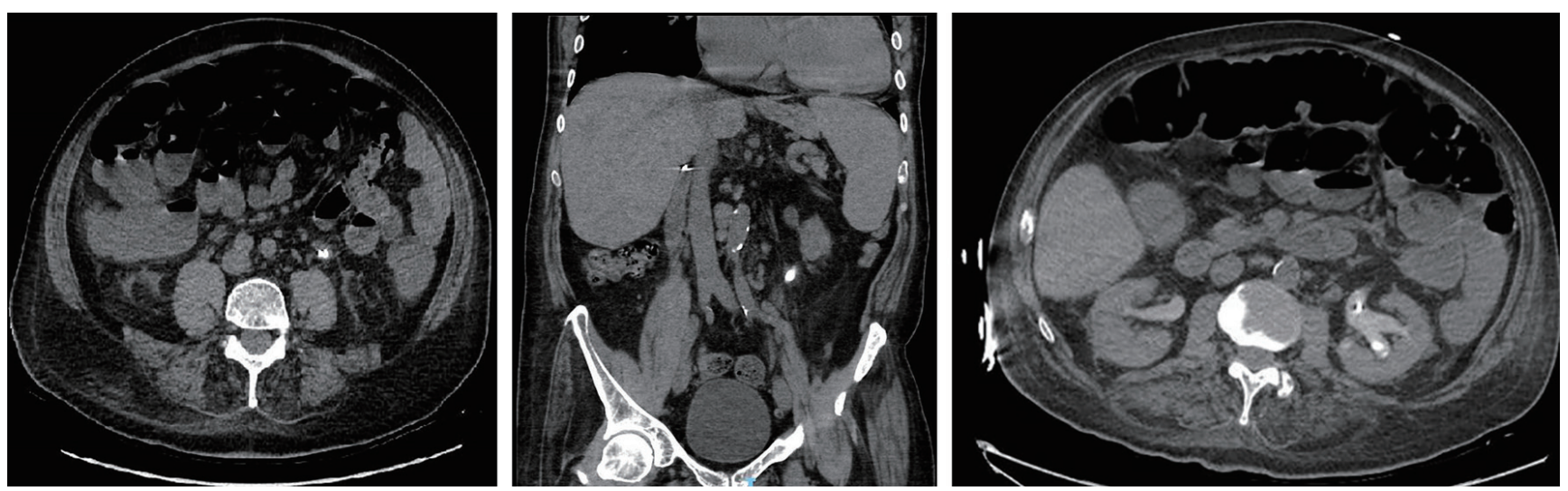

Figure 1. Figure 1. Obstructive stone at the left proximal ureter measuring $2.3 \times 1 \mathrm{~cm}$ with surrounding fat stranding and hydronephrosis in axial and coronal view.

$2.7 \mathrm{mEq} / \mathrm{L}$, phosphorus of $5 \mathrm{mg} / \mathrm{dL}$, magnesium of $1.6 \mathrm{mg} /$ $\mathrm{dL}$, sodium of $148 \mathrm{mEq} / \mathrm{L}$, calcium of $10 \mathrm{mg} / \mathrm{dL}$, lactate dehydrogenase $(\mathrm{LDH})$ of $408 \mathrm{U} / \mathrm{L}$, a borderline elevation in liver function tests and a creatinine of $6.6 \mathrm{mg} / \mathrm{dL}$ with urea of 113 $\mathrm{mg} / \mathrm{dL}$ with a baseline of 1 and a high anion gap of 16 with a bicarbonate level of $15 \mathrm{mEq} / \mathrm{L}$. His calculated glomerular filtration rate (GFR) was $<10$, and he had highly elevated uric acid of $23.5 \mathrm{mg} / \mathrm{dL}$.

The patient was admitted for further management, which primarily included a transthoracic echocardiography. The echo revealed heart failure with preserved ejection fraction and a severe tricuspid valve regurgitation, explaining the patient's lower leg edema found on initial presentation. Also, a bone marrow aspiration cytology and biopsy were done to rule out malignancy. The patient was diagnosed with AML and he was scheduled for induction therapy and started on supportive management and allopurinol prophylactically.

Abdominal and pelvic computed tomography (CT) scan was performed due to pelvic/flank pain but also to further assess the urine analysis (U/A) that showed 80 - 100 red blood count (RBC), 80 - 100 white blood count (WBC) and numerous uric acid crystals. Images came out significant for an obstructive stone in the left proximal ureter measuring $2.3 \times 1 \mathrm{~cm}$ with subsequent left hydronephrosis. Hence the patient underwent JJ catheter insertion (Fig. 1).

On day 1 post-operatively, the patient started complaining of severe left knee pain and swelling. On physical exam, the knee was warm, tender with limited range of motion to $20^{\circ}$ of flexion and extension. Doppler ultrasound of the lower extremities ruled out any occlusion or deep venous thromboembolism. Arthrocentesis was performed and prednisone was initiated. The synovial fluid analysis contained 11,200 WBCs, proving an inflammatory process, with numerous uric acid crystals, establishing the diagnosis of gout. The patient was therefore treated with colchicine on top of the prednisone, allopurinol, hydration and pain management. At a 1-month follow-up visit, the patient had a normalization in his kidney function with a creatinine level of $1.1 \mathrm{mg} / \mathrm{dL}$ and normal phosphorus level, normal potassium level, hypomagnesemia and a normal level of calcium, LDH trending down to normal range and a uric acid of $3.8 \mathrm{mg} / \mathrm{dL}$.
The patient continue to follow up with oncology team for his leukemia.

\section{Discussion}

This patient with progressively increasing fatigue was diagnosed with AML. Even though his transesophageal echocardiography (TTE) findings were able to identify causes for his symptoms and leg swelling, it was not enough to establish a diagnosis.

Over a few days, the patient had an abrupt increase in his WBCs as a manifestation of his primary disease, which led to a massive elevation in serum uric acid levels. The abnormal kidney function resulted in inability to adequately excrete the excess uric acid. This led to the accumulation of urate in the urinary tract causing the uric acid stone and also painful arthritis.

Studies have shown an association between uric acid and AML prognosis. Researchers studied uric acid as a marker for the severity of AML, which was found to rise with increase in staging of the disease. A low level was associated with a better prognosis [7]. Hereafter looking at the uric acid level is a cost-effective method that helps physicians in managing their leukemic patients. Understanding the extension of the disease and decreasing the prevalence of encountering previously mentioned complications can improve patients' disease course and chances of remission.

In this case, the patient had no previous uric acid level, which prevented us from saying that the gout attack was solely the result of an acute hyperuricemia secondary to AML and not from a prior predisposition. To note that the patient did not have the complete picture of tumor lysis syndrome, since he only had hyperuricemia and hyperphosphatemia.

This report is of high importance because it describes a case of AML in an adult that presents with not only one complication of hyperuricemia but two in a very short time period.

It highlights the importance of rapid diagnosis and management of acute leukemia. The delay in treatment can cause major complications such as tumor lysis syndrome, or mild one, such as hyperuricemia alone. Also, management should be aimed at 
lowering uric acid levels to prevent acute joint arthritis.

\section{Conclusions}

From the literature and the case presented, we deduct that acute leukemia can present with the abnormalities in biochemistries, from which we should always look closely at the uric acid level to prevent complications. In some cases, when hyperuricemia is found, it is acceptable to have an aggressive approach in treatment, at the benefit of improving prognosis.

\section{Acknowledgments}

None to declare.

\section{Financial Disclosure}

This case report was not funded.

\section{Conflict of Interest}

The authors declare no conflict of interest.

\section{Informed Consent}

The patient was contacted, and a written consent was taken to write this case report and to share the figures.

\section{Author Contributions}

Cima Hamieh: conception and design of study and drafting the manuscript. Mahmoud El-Hussein: conception and design of study and drafting the manuscript. Elie Zaghrini: revising the manuscript critically for important intellectual content.

\section{Data Availability}

The authors declare that data supporting the findings of this study are available within the article.

\section{Abbreviations}

AML: acute myeloid leukemia; PND: paroxysmal nocturnal dyspnea; MCV: mean corpuscular volume; GFR: glomerular filtration rate; $\mathrm{CT}$ : computed tomography; U/A: urine analysis; RBC: red blood cell; WBC: white blood cell; LDH: lactate dehydrogenase

\section{References}

1. Cancer C. n.d.). Hyperuricemia (High Uric Acid). Retrieved October 05, 2020, from: http://chemocare.com/chemotherapy/side-effects/hyperuricemia-high-uric-acid.aspx.

2. Blanton FM. Acute gout associated with chronic myelogenous leukemia. Ann Intern Med. 1958;48(5):11181121.

3. Choi DH, Lee HS. A case of gouty arthritis following percutaneous radiofrequency ablation for hepatocellular carcinoma. World J Gastroenterol. 2010;16(6):778-781.

4. Anonymous. (2015, February 26). Side Effects. Retrieved October 05, 2020, from: https://www.llscanada.org/leukemia/acute-lymphoblastic-leukemia/treatment/side-effects.

5. Daly GF, Barnard EB, Thoreson L. Renal Calculi: An Unusual Presentation of T-Cell Acute Lymphoblastic Leukemia. Pediatrics. 2016;137(1).

6. Jaing TH, Hung IJ, Lin CJ, Chiu CH, Luo CC, Wang CJ. Acute myeloid leukemia complicated with staghorn calculus. Jpn J Clin Oncol. 2002;32(9):365-367.

7. Yamauchi T, Negoro E, Lee S, Takai M, Matsuda Y, Takagi $\mathrm{K}$, Kishi S, et al. A high serum uric acid level is associated with poor prognosis in patients with acute myeloid leukemia. Anticancer Res. 2013;33(9):3947-3951. 